\title{
Urban vehicular traffic: Fitting the data with a hybrid stochastic model. Part II
}

\author{
A. Amadio ${ }^{a}$, F. Nicuesa ${ }^{a}$, D. Otero ${ }^{\text {a }}$, D. Galetti ${ }^{\text {b }}$, S.S. Mizrahi ${ }^{\text {c,* }}$ \\ a Facultad Regional General Pacheco, Universidad Técnica Nacional (UTN), 1617, Buenos Aires, Argentina \\ b Instituto de Física Teórica, Universidade Estadual Paulista (UNESP), 01140-070, São Paulo, SP, Brazil

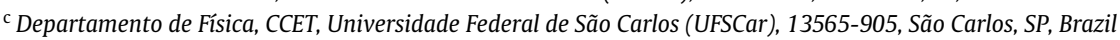

\section{H I G H L I G H T S}

- We use the theory and models developed in Part I: Physica A 509 (2018) 97-110.

- The data originate from a sector of Tigre, Buenos Aires Province, Argentina.

- The traffic flow is described by a hybrid model: 2(linear) and 3(nonlinear) from Part I.

- Our analysis suggests the traffic flow could be optimized by changing some current rules.

- The goals were convincingly achieved as the hybrid model fits well within the error bars.

\section{A R T I C L E I N F O}

\section{Article history:}

Received 23 March 2018

Received in revised form 30 March 2018

Available online 15 June 2018

\section{Keywords:}

Urban traffic

Stochastic process

Data analysis

Hybrid model

\begin{abstract}
A B S T R A C T
In this second part of our research we used the models presented in Modeling vehicular traffic networks. Part I (Otero et al., 2018) to perform an analysis of the urban traffic as recorded by cameras distributed in a chosen sector of Tigre, a city localized in the province of Buenos Aires, Argentina. We found that the circulation of vehicles - the traffic dynamics -, along a whole day, can be described by a hybrid model that is an adapted blend of model 2 , for an open linear system, with model 3, which is nonlinear, developed in Part I. The objectives of this work were, firstly, to verify whether the vehicular flux can be modeled as an $n$-step stochastic process for its evolution, $n$ for the time. Secondly, to find out if the model, with its parameters fixed to describe the traffic of a single day, may adequately describe the traffic in other days. Thirdly, to propose changes in the already established set of the urban traffic rules in order to optimize the vehicular flow and to diminish the average time that a vehicle stays idle at the semaphores. We estimate that the goals were achieved satisfactorily within the margins of the experimental errors of the collected data.
\end{abstract} (C) 2018 Elsevier B.V. All rights reserved.

\section{Introduction}

In a preceding paper, to be referred as Part I (Modeling vehicular traffic networks. Part I [1]), we developed three models for the description of networks representing the vehicular traffic between several sites that could be cities, parking lots, car

\footnotetext{
* Corresponding author.

E-mail addresses: ariel.amadio.aaa@gmail.com (A. Amadio),n_facundo@hotmail.com (F. Nicuesa), dinootero@fibertel.com.ar (D. Otero), galetti@ift.unesp.br (D. Galetti), salomon@df.ufscar.br (S.S. Mizrahi).
} 

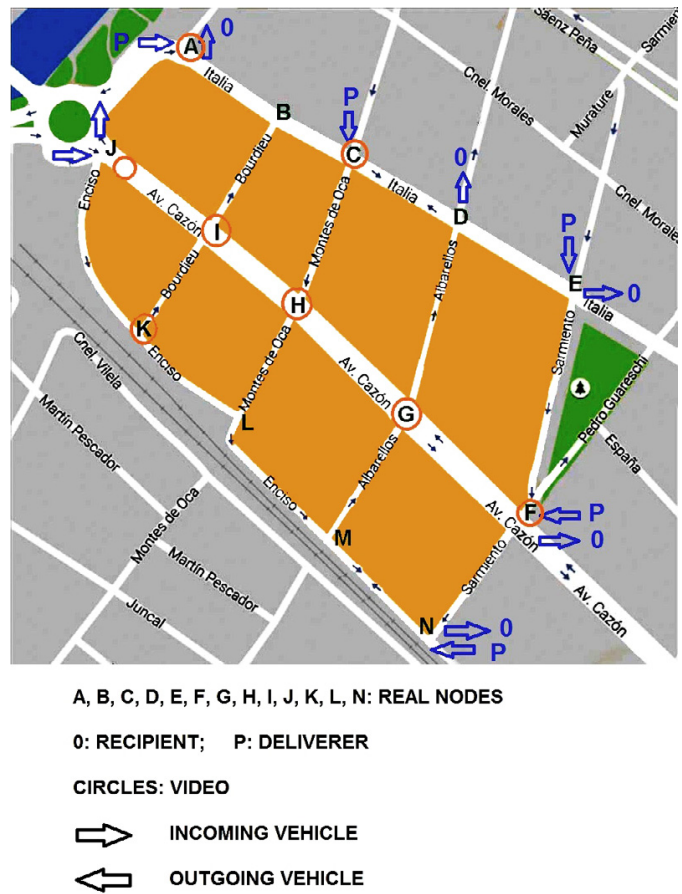

Fig. 1. Chosen sector in downtown of Tigre. It consists of fourteen intersections, with cameras installed in eight of them (identified by the orange circles) for recording the traffic flow.

rental agencies, etc.... A network is composed by arteries, intersections and blocks; pictorially, it is represented by a digraph made of vertices and directed edges. Mathematically the digraph is pictured as a matrix, whose diagonal entries represent the number of vehicles at the sites (vertices) and the off-diagonal ones stand for the number of vehicles in transit, from one site to another, along the arteries (edges). By normalizing each row, one gets a stochastic matrix, whose entries are numbers in the interval $[0,1]$. The evolution of a vehicular distribution at time $n$ is obtained step by step: one multiplies the initial vector, whose components stand for the distribution of vehicles within the network at $n=0$, by the stochastic matrix raised to the power $n$. This procedure is admitted to be adequate to describe the behavior of the traffic dynamics, as long as that matrix has been constructed confidently. In Part I we have presented a theoretical description, with numerical examples, of a finite network with linear and nonlinear models for closed and open systems. In the present Part II we used the methods and models of Part I to analyze the collected data for the traffic of vehicles in a chosen sector of the city of Tigre, Province of Buenos Aires, Argentina, see Fig. 1. The detailed formalism is presented in [1] and in the therein references.

We constructed two stochastic matrices, $\mathbb{M}^{(1)}$ and $\mathbb{M}^{(2)}$, one for each period of a particular day (from 06:00 AM to 08:00 PM and from 08:00 PM to 06:00 AM of the following day), that when evolved, according to the proposed model, permitted to verify that the observed vehicular distribution is reproduced satisfactorily within the experimental error of the data. Even for other days the vehicular distributions were adequately described using the very same matrices $\mathbb{M}^{(1)}$ and $\mathbb{M}^{(2)}$.

Since the vertices of a digraph represent street intersections in the city map, we will also refer to them as nodes. We shaped the dynamics of the influx and outflux of vehicles by choosing conveniently the entries of $\mathbb{M}^{(1)}$ and $\mathbb{M}^{(2)}$. We found that a hybrid model that is an adapted merging of the linear open system model with the nonlinear one, introduced in the Part I, produces numerical results that are in fair agreement with the data from the traffic flow. The data we used came from samples counts although the traffic was continuously monitored during $24 \mathrm{~h}$.

The traffic in a city, and even in one of its sectors, is not uniform during a whole 24-h day, instead it varies continuously. In our chosen sector we verified that for an usual working day the influx begins to increase steadfastly after 6:00 AM. Then, from 10:00 AM to 8:00 PM it stabilizes with some oscillations, that we call a quasi-stationary régime, which is due to the commercial and social activities, namely, the opening and closing of businesses, shops, offices, hospitals, police station and schools within the perimeter of the network. Afterward it decreases rapidly, the vehicular outflux starts at 8:00 PM (when the activities reduce at the end of the day) and continues until 4:00 AM of the following day. From 4:00 AM to 6:00 AM there is practically no circulation of vehicles. We are interested in modeling this dynamics.

The selected sector of the city, see Fig. 1, has several blocks separated by streets and avenues, where the arrows indicate the directions of the vehicular flux. Although the cameras were positioned at only eight intersections, labeled $A, C, F, G, H, I, J, K$ and marked by the orange circles, we have included six additional intersections, thus resulting in a network of fourteen intersections. The roads and avenues that link them are referred by their names. This sector has a 


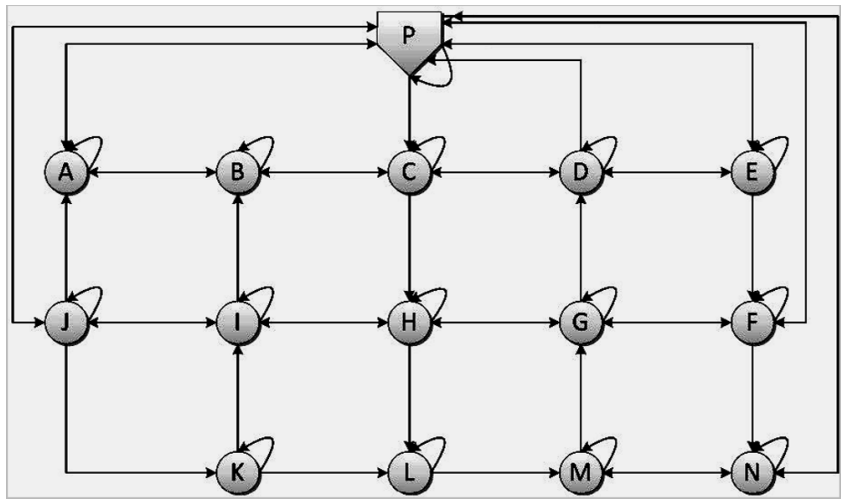

Fig. 2. Digraph picturing the network. The node $P$ does not represent a real intersection, it is an extra auxiliary node (a reservoir) accounting for the influx and outflux of vehicles within the network. All other nodes represent the arteries intersections. Each of them has a loop to which one associates the number of vehicles standing idle at red lights or parked along the sidewalks.

reduced number of entries and exits, and still conveys a heavy traffic during the day without the occurrence of significant traffic jam. The heavier traffic occurs in Cazón avenue, because it is the artery where most businesses and public offices are located. Then comes Italia avenue, whereas the other arteries have comparatively less intense traffic. This network is an open system, because at the nodes $A, C, E, F, N$ and $J$ the vehicles arrive from outside the circuit (an influx of vehicles), while at nodes $A, D, E, F, N$ and $J$ there is a drain of vehicles (an outflux). According to the observed characteristics of the traffic, we are modeling the system assuming that, basically, the vehicles circulating within the network arrive at and depart through the nodes, with the exception of a small number that are parked along the sidewalks nearby the intersections plus those standing idle awaiting for the semaphores to change light. An extra auxiliary node $P$ was added to the circuit, which assumes the rôle of a reservoir, it represents a locus of influx and outflux of vehicles for the network, see Fig. 1 (map) and 2 (digraph). Recently, another model [2], quite different from ours, was proposed with the same aim, to describe the vehicular traffic flow.

\section{Hybrid model for vehicular traffic}

The evolution of the average distribution of vehicles in our network is represented by the vector $\mathbf{v}^{\dagger}(n)$, with $n$ a positive integer, standing for discretized time, as

$$
\mathbf{v}^{\dagger}(n)=\mathbf{v}_{0}^{\dagger} \mathbb{M}_{e f f}(n)
$$

where each component of $\mathbf{v}^{\dagger}(n)$ is the vehicular distribution at one node plus those in transit to other nodes, at time $n$, and $\mathbf{v}_{0}^{\dagger}$ stands for the initial distribution. The effective matrix is the product of $n+1$ matrices,

$$
\mathbb{M}_{\text {eff }}(n)=\mathbb{E}(n) \mathbb{M}^{n}
$$

The matrix $\mathbb{M}$ is stochastic and its entries stand for the initial conditions for the evolution; the matrix $\mathbb{E}(n)$ contains the effects of the environment, because the vehicular traffic is an open system. $\mathbb{E}(n)$ is not stochastic and we call it the environment influence matrix. The effective matrix (2) is the generator of the evolution of $\mathbf{v}_{0}^{\dagger}$. In short, the evolution of an open nonlinear network is based on a hybrid of two models and the vehicular distribution $\mathbf{v}^{\dagger}(n)$ is also nonlinear as the sum of its components changes with $n$. As $\mathbb{M}$, the matrix $\mathbb{E}(n)$ is also a square matrix of dimension $N$, that we defined as

$$
\mathbb{E}(n)=\left(\begin{array}{cccccc}
1 & 0 & \cdots & 0 & 0 & 0 \\
& 1 & \cdots & 0 & 0 & 0 \\
\cdots & \cdots & \ddots & 0 & \cdots & \cdots \\
0 & 0 & \cdots & 1 & 0 & 0 \\
0 & 0 & \cdots & 0 & 1 & 0 \\
0 & 0 & \cdots & 0 & 0 & 1+\frac{f(n)}{\mathcal{N}}
\end{array}\right),
$$

where $f(n)$ is a specific function and $\mathcal{N}$ is the total number of vehicles within the virtual reservoir $P$. The parameters are adjusted by minimizing the $\chi^{2}$ deviations from the collected data. We outline the network as the array in Table 1 , where the numbers for non-zero entries are set either by the counts of vehicles, or by estimation for the intersections without cameras. The zeros stand for the wrong ways for the vehicles. 
Table 1

The entries $p_{i j}$ are for the number of vehicles at vertices and edges associated with the digraph 2 that represents the chosen city sector shown in Fig. 1.

\begin{tabular}{|c|c|c|c|c|c|c|c|c|c|c|c|c|c|c|c|}
\hline & $A$ & $B$ & $C$ & $D$ & $E$ & $F$ & $G$ & $H$ & $I$ & $J$ & $K$ & $L$ & $M$ & $N$ & $P$ \\
\hline$A$ & $p_{A A}$ & $p_{A B}$ & 0 & 0 & 0 & 0 & 0 & 0 & 0 & $p_{A J}$ & 0 & 0 & 0 & 0 & $p_{A P}$ \\
\hline$B$ & $p_{B A}$ & $p_{B B}$ & $p_{B C}$ & 0 & 0 & 0 & $p_{B G}$ & 0 & 0 & 0 & 0 & 0 & 0 & 0 & 0 \\
\hline$C$ & 0 & $p_{C B}$ & $p_{C C}$ & $p_{C D}$ & 0 & 0 & 0 & $p_{C H}$ & 0 & 0 & 0 & 0 & 0 & 0 & 0 \\
\hline$D$ & 0 & 0 & $p_{D C}$ & $p_{D D}$ & $p_{D E}$ & 0 & $p_{D G}$ & 0 & 0 & 0 & 0 & 0 & 0 & 0 & $p_{D P}$ \\
\hline$E$ & 0 & 0 & 0 & $p_{E D}$ & $P_{E E}$ & $p_{E F}$ & 0 & 0 & 0 & 0 & 0 & 0 & 0 & 0 & $p_{E P}$ \\
\hline$F$ & 0 & 0 & 0 & 0 & 0 & $p_{F F}$ & $p_{F G}$ & 0 & 0 & 0 & 0 & 0 & 0 & $p_{F N}$ & $p_{F P}$ \\
\hline$G$ & 0 & 0 & 0 & $p_{G D}$ & 0 & $p_{G F}$ & $p_{G G}$ & $p_{G H}$ & 0 & 0 & 0 & 0 & 0 & 0 & 0 \\
\hline$H$ & 0 & 0 & 0 & 0 & 0 & 0 & $p_{H G}$ & $p_{H H}$ & $p_{H I}$ & 0 & 0 & $p_{H L}$ & 0 & 0 & 0 \\
\hline$I$ & 0 & $p_{I A}$ & 0 & 0 & 0 & 0 & 0 & $p_{I H}$ & $p_{I I}$ & $p_{I J}$ & 0 & 0 & 0 & 0 & 0 \\
\hline$J$ & $p_{J A}$ & 0 & 0 & 0 & 0 & 0 & 0 & 0 & $p_{J I}$ & $p_{J J}$ & $p_{J K}$ & 0 & 0 & 0 & $p_{J P}$ \\
\hline$K$ & 0 & 0 & 0 & 0 & 0 & 0 & 0 & 0 & $p_{K I}$ & 0 & $p_{K K}$ & $p_{K L}$ & 0 & 0 & 0 \\
\hline$L$ & 0 & 0 & 0 & 0 & 0 & 0 & 0 & 0 & 0 & 0 & 0 & $p_{L L}$ & $p_{L M}$ & 0 & 0 \\
\hline$M$ & 0 & 0 & 0 & 0 & 0 & 0 & $p_{M G}$ & 0 & 0 & 0 & 0 & 0 & $p_{M M}$ & $p_{M N}$ & 0 \\
\hline$N$ & 0 & 0 & 0 & 0 & 0 & 0 & 0 & 0 & 0 & 0 & 0 & 0 & $p_{N M}$ & $p_{N N}$ & $p_{N P}$ \\
\hline$P$ & $p_{P A}$ & 0 & $p_{P C}$ & 0 & $p_{P E}$ & $p_{P F}$ & 0 & 0 & 0 & $p_{P J}$ & 0 & 0 & 0 & $p_{P N}$ & $p_{P P}$ \\
\hline
\end{tabular}

\section{Methodology of collecting and analyzing the data}

Our modus operandi consisted in counting cars, vans, trucks, buses, and motorcycles. We adopted a sampling procedure: at each intersection having an installed camera we counted the number of vehicles during six consecutive minutes at every two hours. For example, beginning at 10:00 AM we counted until 10:06 AM, which we adopted as a 5\% sample.

At 6:00 AM and early hours of the morning the number of vehicles in circulation is sparse, so we established the timezero vector as $\mathbf{v}_{0}^{\dagger}=\left(\begin{array}{lllll}0 & 0 & \cdots & 0 & \mathcal{N}\end{array}\right)$, having fifteen components with the first fourteen being null and the fifteenth standing for the number of vehicles in node $P$.

The distances that separate the intersections (block lengths) vary between 120 and $140 \mathrm{~m}$, and we verified that a vehicle takes between $20 \mathrm{~s}$ and $25 \mathrm{~s}$ to go from one intersection to the next. Therefore, we adopted $22.5 \mathrm{~s}$ as the unit of time, i.e. for the transition from one node to the next for the $n$-step stochastic process. The evolution of the vehicular distribution within the network is directly associated with this time. For example, one hour corresponds to 160 time units, thus the matrices (6) and (7) are raised to powers as $\left(\mathbb{M}^{(i)}\right)^{n}, n=1, \ldots, 160$, for their evolution along one hour.

The rate of convergence to the quasi-stationary régime of the distribution of vehicles depends on $n$, and in accordance with each intersection it varies between 300 and 400 . At the quasi-stationary régime the counted vehicles at the nodes fluctuate exceeding the Poisson dispersion. The dispersions are represented by the vertical bars in Figs. 3-10.

We classified the daily data in four periods: (1) From 6:00 AM to 10:00 AM, when the influx starts with an almost zero flow of vehicles, then increasing along the first hours of the day. (2) From 10:00 AM to 08:00 PM the traffic roughly stabilizes, showing oscillations around some average value. The quasi-stationary régime is established and the difference between influx and outflux of vehicles is quite low. (3) From 08:00 PM to 04:00 AM of the next day, the vehicles begin leaving the network and an intense outflux, with almost no influx, is observed. (4) From 04:00 AM to 06:00 AM there is practically no circulation of vehicles within the network.

At the intersections with cameras, the vehicles standing idle at the semaphores or parked along the nearby sidewalks contributed to adjust the diagonal entries of matrices (6) and (7), while the vehicles crossing one intersection or changing direction (left or right) contributed to adjust the non-diagonal entries. At intersections without cameras the entries of the matrices were estimated and the numerical values were set such to get a best fit for the available data. The influx of vehicles within the network goes from node $P$ into the nodes $A, C, E, F, J, N$.

In average, the number of vehicles at one intersection plus those in transit to the other intersections, at time $n$, is given by a component of the vector (1), with matrix (6) on the right hand side: $\mathbf{v}_{1}^{\dagger}(n)=\mathbf{v}_{0}^{\dagger} \mathbb{M}_{\text {eff }}^{(1)}(n)$. Notwithstanding, as the semaphores do not performed in good synchrony we opted to count the vehicles at each node independently of the others, so we projected out each component that was compared with the data. For instance, for node $k$ the average number of vehicles is

$$
v_{1, k}\left(n_{k}\right)=\left(\mathbf{v}_{0}^{\dagger} \mathbb{M}_{\text {eff }}^{(1)}\left(n_{k}\right)\right)\left(\begin{array}{c}
0_{1} \\
\vdots \\
1_{k} \\
\vdots \\
0_{15}
\end{array}\right) .
$$

At the beginning of the third period - 8:00 PM to 04:00 AM - the traffic slows down because the vehicles begin to leave the sector, displaying an outflux from nodes $A, D, E, F, N, J$ and an influx towards the node $P$. The evolution of each node for an 
emptying network goes as

$$
v_{2, k}\left(n_{k}^{\prime}, \bar{n}_{k}\right)=\left(\mathbf{v}_{0}^{\dagger} \mathbb{M}_{e f f}^{(1)}\left(\bar{n}_{k}\right)\right)\left(\mathbb{M}^{(2)}\right)^{n_{k}^{\prime}}\left(\begin{array}{c}
0_{1} \\
\vdots \\
1_{k} \\
\vdots \\
0_{15}
\end{array}\right),
$$

where $\bar{n}_{k}$ is now a fixed parameter, being the last number used for the previous period, whose value varies in the interval (2200, 2300), depending on each node $k$. The time is represented by $n_{k}^{\prime}$, and $\mathbb{M}^{(2)}$ is the generator of the evolution for the third period. Depending on the node it refers, the "last" number $\bar{n}_{k}^{\prime}$ varies in the interval $(1200,1300)$.

From 6:00 AM to 6:06 AM the Poisson dispersion is quite high because vehicles are not observed at some intersections while at others no more than six vehicles are counted (a dispersion around $45 \%$ ). Comparatively, in the quasi-stationary régime the vehicles that cross the intersections of Cazón avenue present an $8 \%$ dispersion.

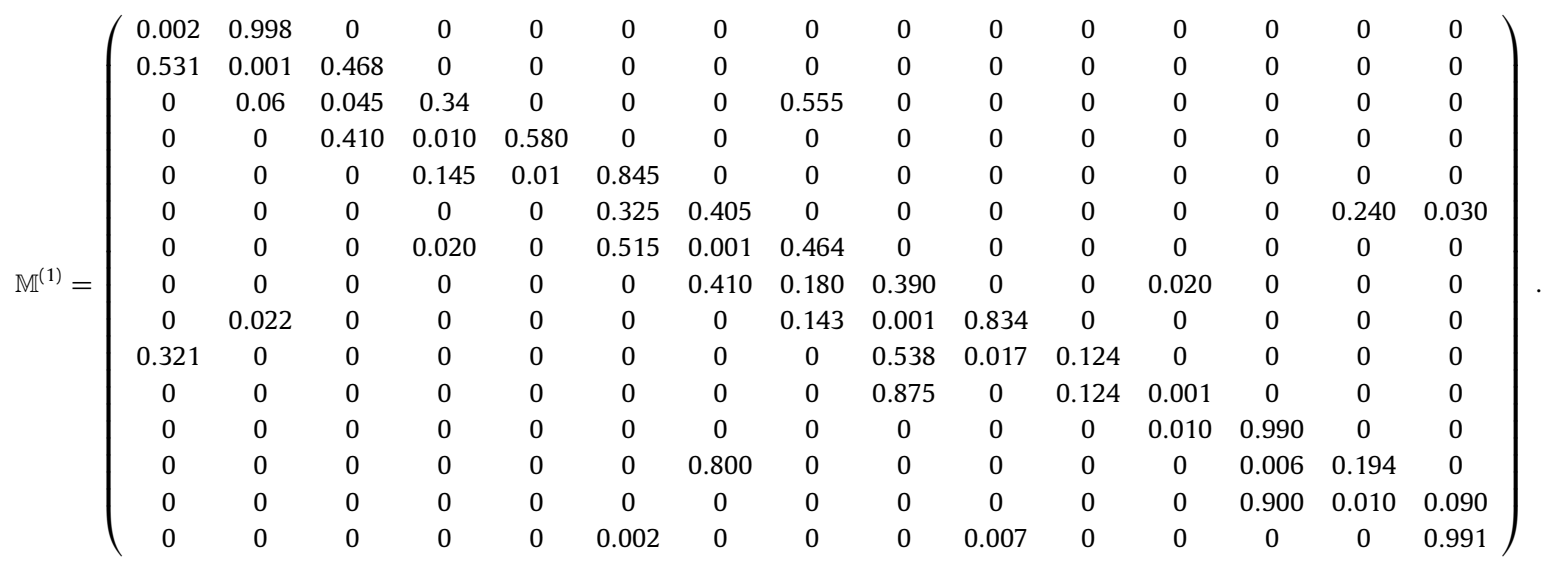

To describe the traffic from 8:00 PM to 4:00 AM, when the outflux of vehicles begins, Eq. (5), we used the matrix (7), whose entries, also fixed by best.

$$
\mathbb{M}^{(2)}=\left(\begin{array}{ccccccccccccccc}
0.003 & 0.997 & 0 & 0 & 0 & 0 & 0 & 0 & 0 & 0 & 0 & 0 & 0 & 0 & 0 \\
0.530 & 0.001 & 0.469 & 0 & 0 & 0 & 0 & 0 & 0 & 0 & 0 & 0 & 0 & 0 & 0 \\
0 & 0.030 & 0.085 & 0.320 & 0 & 0 & 0 & 0.565 & 0 & 0 & 0 & 0 & 0 & 0 & 0 \\
0 & 0 & 0.381 & 0.040 & 0.579 & 0 & 0 & 0 & 0 & 0 & 0 & 0 & 0 & 0 & 0 \\
0 & 0 & 0 & 0.140 & 0.020 & 0.790 & 0 & 0 & 0 & 0 & 0 & 0 & 0 & 0 & 0.050 \\
0 & 0 & 0 & 0 & 0 & 0.344 & 0.392 & 0 & 0 & 0 & 0 & 0 & 0 & 0.260 & 0.004 \\
0 & 0 & 0 & 0.020 & 0 & 0.510 & 0.001 & 0.469 & 0 & 0 & 0 & 0 & 0 & 0 & 0 \\
0 & 0 & 0 & 0 & 0 & 0 & 0.342 & 0.188 & 0.370 & 0 & 0 & 0.100 & 0 & 0 & 0 \\
0 & 0.010 & 0 & 0 & 0 & 0 & 0 & 0.045 & 0.001 & 0.944 & 0 & 0 & 0 & 0 & 0 \\
0.320 & 0 & 0 & 0 & 0 & 0 & 0 & 0 & 0.469 & 0.090 & 0.120 & 0 & 0 & 0 & 0.001 \\
0 & 0 & 0 & 0 & 0 & 0 & 0 & 0 & 0.875 & 0 & 0.124 & 0.001 & 0 & 0 & 0 \\
0 & 0 & 0 & 0 & 0 & 0 & 0 & 0 & 0 & 0 & 0 & 0.010 & 0.990 & 0 & 0 \\
0 & 0 & 0 & 0 & 0 & 0 & 0.800 & 0 & 0 & 0 & 0 & 0 & 0.006 & 0.194 & 0 \\
0 & 0 & 0 & 0 & 0 & 0 & 0 & 0 & 0 & 0 & 0 & 0 & 0.960 & 0.010 & 0.030 \\
0 & 0 & 0 & 0 & 0 & 0 & 0 & 0 & 0 & 0 & 0 & 0 & 0 & 0 & 1
\end{array}\right) .
$$

The numerical entries of matrices $\mathbb{M}^{(1)}$ and $\mathbb{M}^{(2)}$ were obtained by minimizing $\chi^{2}$, for influx and outflux, to get the best fit [3],

$$
\chi_{\text {influx }}^{2}=\sum_{j=1}^{8} \sum_{k=1}^{8} \frac{\left(v_{1, k, j}^{o b s}-v_{1, k, j}^{\text {theor }}\right)^{2}}{v_{1, k, j}^{o b s}},
$$




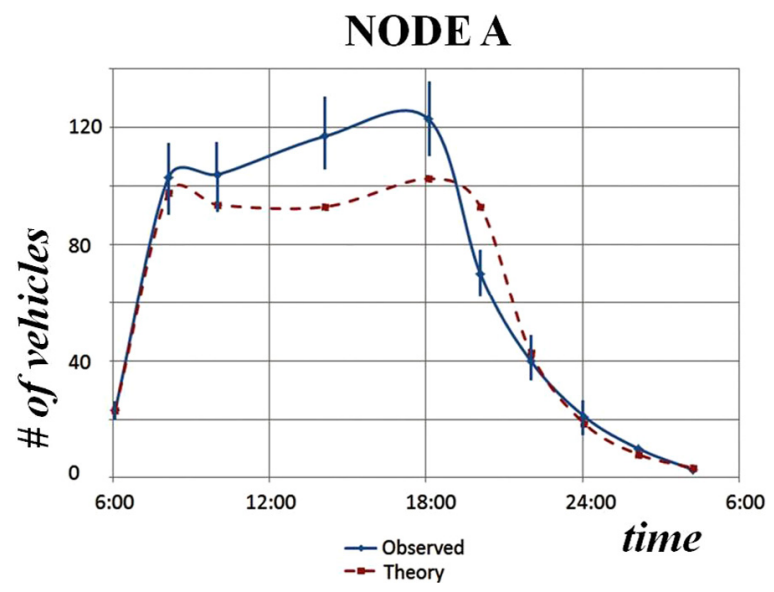

Fig. 3. Variation of the flux (number of vehicles for every six minutes) going through the node $A$ along $22 \mathrm{~h}$, beginning nearby 8:00 AM until 4:00 AM of the next day. Below the abscissa, the time in conventional notation. The error bars represent the dispersion of the empirical data collected in May $18,2016$. The hybrid model produces the dashed curve in red.

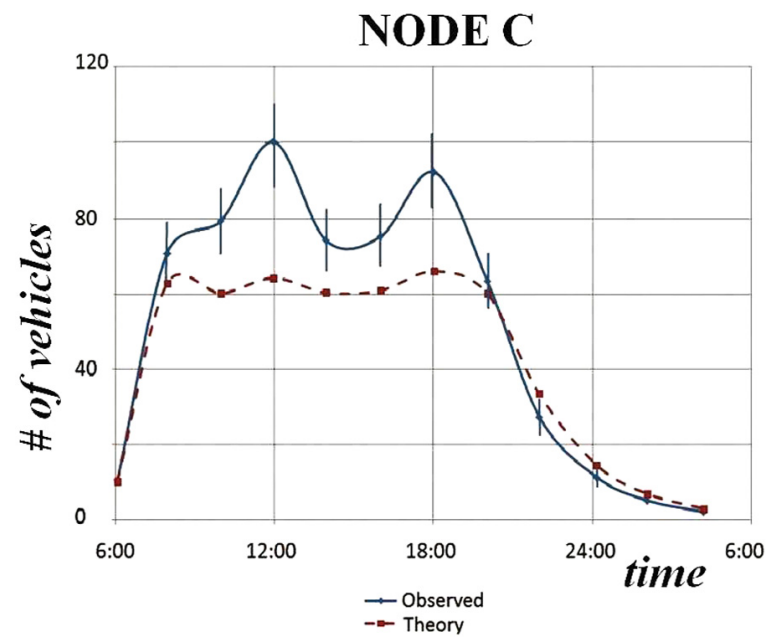

Fig. 4. Variation of the flux (number of vehicles for every six minutes) going through the node $C$ along $22 \mathrm{~h}$, beginning nearby 8:00 AM until 4:00 AM of the next day. Below the abscissa, the time in conventional notation. The error bars represent the dispersion of the empirical data collected in May $18,2016$. The hybrid model produces the dashed curve in red.

and

$$
\chi_{\text {outflux }}^{2}=\sum_{j=1}^{8} \sum_{k=1}^{4} \frac{\left(v_{2, k, j}^{\text {obs }}-v_{2, k, j}^{\text {theor }}\right)^{2}}{v_{2, k, j}^{\text {obs }}},
$$

where the subscript $j$ stands for the eight intersections with cameras and $k$ for the six minutes sample at every two hours. For the influx counting we had 62 effective observations out from the 64 possible ones, while for the outflux all 32 observation were effective. For the influx transient and the quasi-stationary régime (06:00 AM to 08:00 PM) we have considered: (1) the normalization of each row of the matrix and, (2) the effects of the environment on the network, namely, in Eq. (3) we set $\mathcal{N}=1970$ and the most adequate function is

$$
f(n)=(20.0+0.0576 n) \cos \left(\frac{\pi n}{275.0+0.055 n}\right),
$$

which depicts the oscillations with varying amplitudes and periods. At the nodes where the traffic was monitored the experimental fluxes and those resulting from the hybrid model are shown in Figs. 3-10. The vertical axis stands for the number of vehicle counted every six minutes and in the horizontal axis the time considering $22.5 \mathrm{~s}$ as a unit. The experimental data are from three different days: May 18, 2016 (blue solid line and error bars in the graphs) present in all the eight nodes, Figs. 3-10. The data from April 30 and May 02, 2016 (green error bars in the graphs) are for the nodes $F, G, I$ and $J$, shown in 


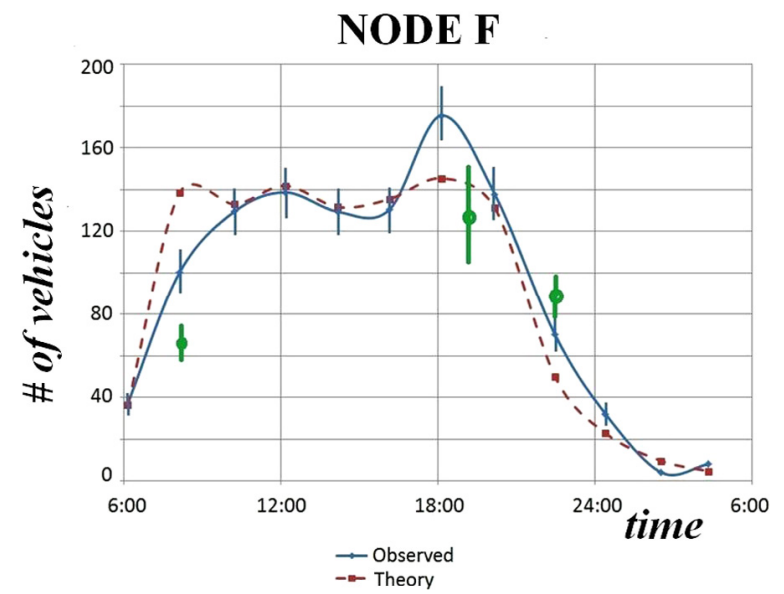

Fig. 5. Variation of the flux (number of vehicles for every six minutes) going through the node $F$ along 22 h, beginning nearby $8: 00$ AM until $4: 00$ AM of the next day. Below the abscissa, the time in conventional notation. The error bars, in blue, represent the dispersion of the empirical data collected in May 18, 2016. The error bars, in green, represent the data collected in April 30, 2016. The hybrid model produces the dashed curve in red. (For interpretation of the references to color in this figure legend, the reader is referred to the web version of this article.)

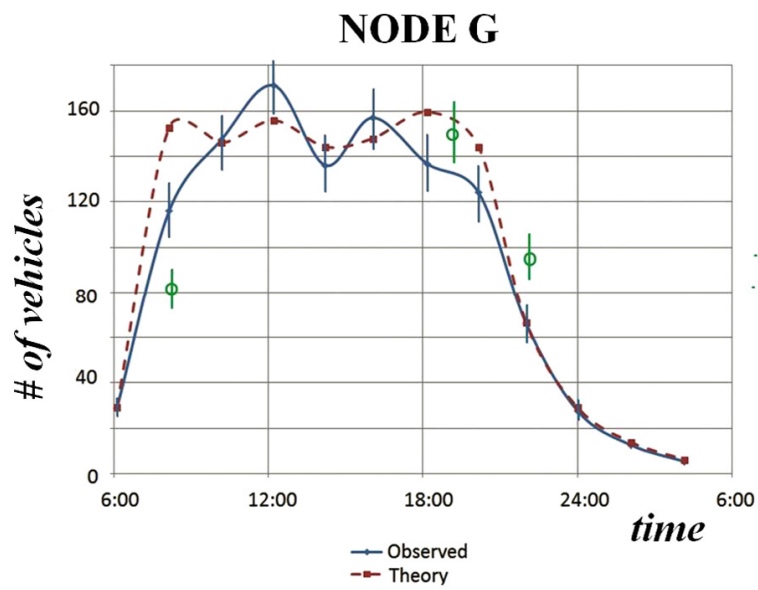

Fig. 6. Variation of the flux (number of vehicles for every six minutes) going through the node $G$ along $22 \mathrm{~h}$, beginning nearby $8: 00$ AM until $4: 00$ AM of the next day. Below the abscissa, the time in conventional notation. The error bars, in blue, represent the dispersion of the empirical data collected in May 18,2016 . The error bars, in green, represent the data collected in April 30, 2016. The hybrid model produces the dashed curve in red. (For interpretation of the references to color in this figure legend, the reader is referred to the web version of this article.)

Figs. 5, 6, 8 and 9. The data from May 19, 2016 (yellow error bars in the graphs) are for the node I only, shown in Fig. 8. The blue solid curve that links the experimental points were obtained by interpolation using the spreadsheet Microsoft Excel. The curves resulting from our model are represented by red dashed lines. In the quasi-stationary régime the hybrid model presents oscillations with, in general, three resolved peaks, although the empirical data show two or three peaks. Nevertheless the curves of the hybrid model stand for the best fit for the traffic flux represented by the distribution of vehicles at all the nodes. Our assessment is that the model and the theoretical approach we embraced describe satisfactorily the traffic complexity within our chosen urban sector.

\section{Additional analysis}

As one of our objectives is to know the number of vehicles circulating through the arteries of the city sector at different moments of the day, we adopted $\mathbb{M}^{(1)}$ and $\mathbb{E}(1)$ as the time-zero matrices, i.e., when the vehicular influx begins. In Fig. 11 we display the evolution of the traffic flux calculated as the number of vehicles per six minutes along the considered arteries. The dots in the figure stand for $\mathbf{v}_{1}\left(n_{0} j\right)\left(n_{0}=320\right)$ with $j=1,2, \ldots, 6$ for times 8:00 AM, 10:00 AM, 12:00 AM, 02:00 PM, 04:00 PM, 06:00 PM and $\mathbf{v}_{2}\left(n_{0}^{\prime} j\right)\left(n_{0}^{\prime}=320\right)$ and $j=1,2, \ldots, 5$ for times 08:00 PM, 10:00 PM, 12:00 PM, 02:00 AM, 04:00 AM.

We hold that modeling serves to assist the traffic controllers by proposing procedures for optimizing the vehicular circulation by diminishing the probability of jams, car accidents and reducing the average travel time between two locations. 


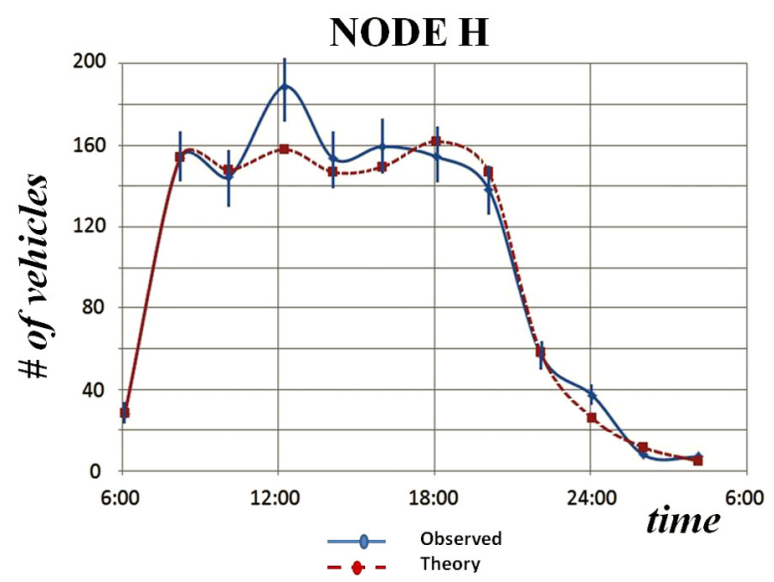

Fig. 7. Variation of the flux (number of vehicles for every six minutes) going through the node $H$ along $22 \mathrm{~h}$, beginning nearby 8:00 AM until 4:00 AM of the next day. Below the abscissa, the time in conventional notation. The error bars represent the dispersion of the empirical data collected in May $18,2016$. The hybrid model produces the dashed curve in red.

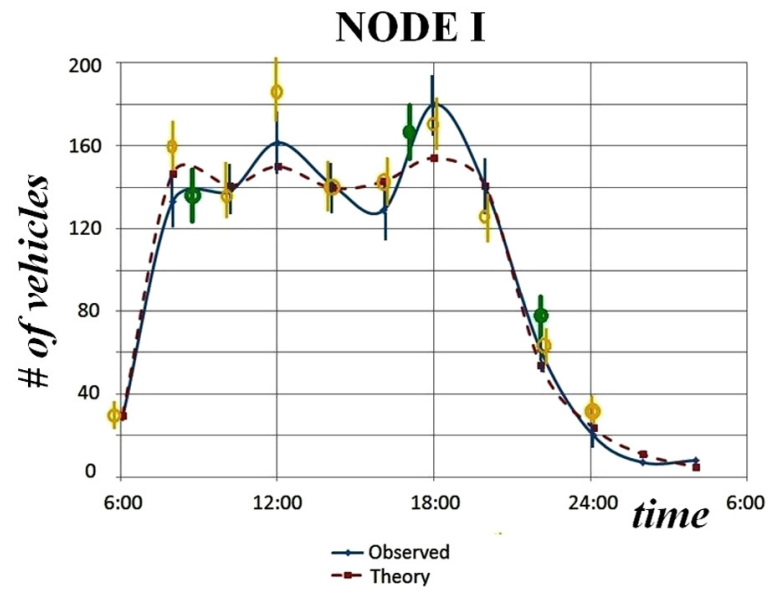

Fig. 8. Variation of the flux (number of vehicles for every six minutes) going through the node $I$ along $22 \mathrm{~h}$, beginning nearby 8:00 AM until 4:00 AM of the next day. Below the abscissa, the time in conventional notation. The error bars, in blue, represent the dispersion of the empirical data collected in May 18,2016 . The error bars, in green, represent the data collected in May 02, 2016. The error bars, in yellow, represent the data collected in May 19, 2016. The hybrid model produces the dashed curve in red. (For interpretation of the references to color in this figure legend, the reader is referred to the web version of this article.)

In particular, a model study can also recommend how to better set the timing of the semaphores lights in the intersections, even in those where cameras are not installed, and to synchronize conveniently their delay times along the main arteries.

Cazón, a two-way avenue, possesses semaphores and cameras at the five intersections comprised in the chosen sector. The green light duration at each intersection is $72 \mathrm{~s}$ while the red light stays on for $38 \mathrm{~s}$. As the considered segment of Cazón avenue has a length of $520 \mathrm{~m}$, by starting at intersection $J$ until crossing $F$ a vehicle could run through it, ideally, in $\approx 47 \mathrm{~s}$ at an average speed of $40 \mathrm{~km} / \mathrm{h}(\approx 11.1 \mathrm{~m} / \mathrm{s})$, without stopping at a red light. Nevertheless, what was observed is that within the period from 10:00 AM to 08:00 PM (the quasi-stationary régime), it takes around $100 \mathrm{~s}$ for a vehicle to go along that section with a unique stop at a red light. So the effective time for a vehicle to run along Cazón goes from the ideal $47 \mathrm{~s}$ to the real $\approx 100 \mathrm{~s}$, which is due to the jam caused by buses stopping at bus stops. So the average speed of a running vehicle is reduced to $30 \mathrm{~km} / \mathrm{h}$, and considering the idle time at one red light, this effectively goes down to $\approx 19 \mathrm{~km} / \mathrm{h}(5.2 \mathrm{~m} / \mathrm{s})$.

Even so, an improvement in the traffic circulation can be made through a simple analysis. At the quasi-stationary régime we considered the ratios for the flux of vehicles for three major adjacent intersecting streets of Cazón: I (for Cazón-Bordieu), $H$ (for Cazón - Montes de Oca) and G (for Cazón - Albarellos),

$$
R_{\alpha}=\frac{\text { Flux of vehicles along Cazón }}{\text { Flux of vehicles along } \alpha},
$$

where $\alpha$ can be $I, H$ or $G$. In average, the calculated numerical results are $R_{I} \approx 2.7, \quad R_{H} \approx 3.2, \quad R_{G} \approx 3.7$, that can also be estimated from the curves in Fig. 11. With the aim of improving the traffic circulation along Cazón avenue and without much 


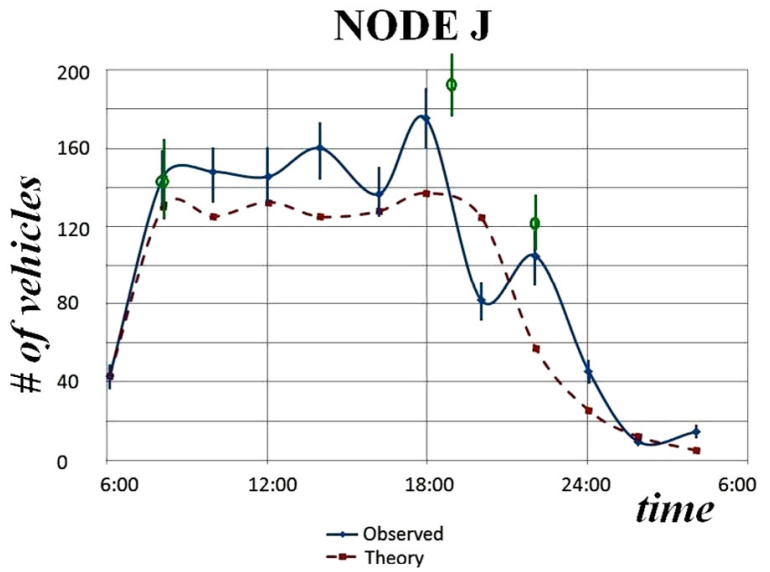

Fig. 9. Variation of the flux of vehicles (number for every six minutes) going through the node $J$ along $22 \mathrm{~h}$, beginning nearby $8: 00$ AM until 4:00 AM of the next day. Below the abscissa, the time in conventional notation. The error bars, in blue, represent the dispersion of the empirical data collected in May 18, 2016. The error bars, in green, represent the data collected in May 02, 2016. The hybrid model produces the dashed curve in red. (For interpretation of the references to color in this figure legend, the reader is referred to the web version of this article.)

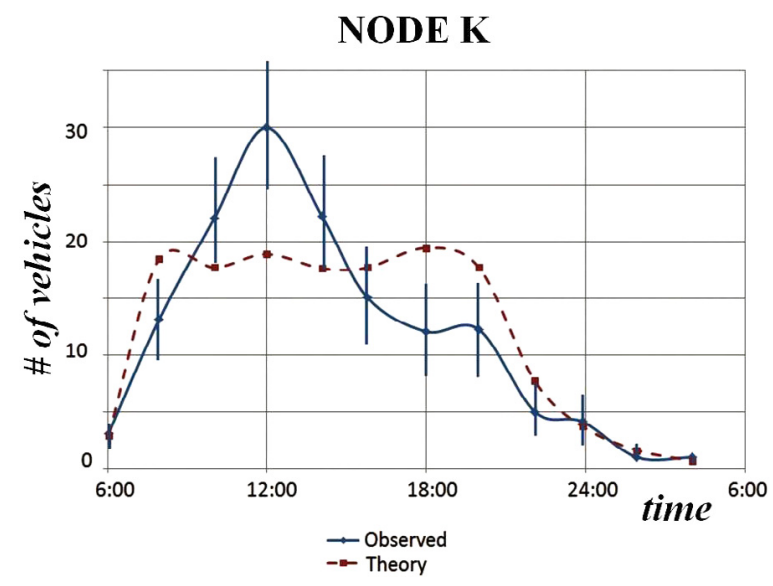

Fig. 10. Variation of the flux (number of vehicles for every six minutes) going through the node $K$ along 22 h, beginning nearby $8: 00$ AM until 4:00 AM of the next day. Below the abscissa, the time in conventional notation. The error bars represent the dispersion of the empirical data collected in May 18,2016 . The hybrid model produces the dashed curve in red.

impairment to the traffic flux of the intersecting arteries, we have assumed the lowest ratio $R=2.7$ for all three intersections. The measured ratio of times for which the semaphore lights remain green is $T_{\text {Cazón }} / T_{\text {intersection }}=72 \mathrm{~s} / 38 \mathrm{~s} \approx 1.9$. Considering $R=2.7$, our model hints to an improvement of the circulation if the traffic controller keeps the $72 \mathrm{~s}$ green light time for Cazón avenue and reduces the green light time for the intersections to $72 / 2.7 \approx 27 \mathrm{~s}$. The reduction from $38 \mathrm{~s}$ to $27 \mathrm{~s}$ for one single stop at a red light in Cazón provides around 30\% economy of fuel consumption of an idle vehicle, permitting thus a significant reduction of $\mathrm{CO}_{2}$ and pollutants emissions, besides benefiting drivers and passengers with a gain in time.

\section{Summary and conclusions}

In Part I of our research [1], we have proposed three models that describe ideally the traffic flow. In the present Part II we used the proposed models to analyze and emulate a real situation for the urban traffic. We counted the number of vehicles from tapes disposed to us by the traffic controllers, regarding the circulation of vehicles in that particular sector of the city, shown in Fig. 1. The recording cameras were disposed at eight intersections. The data were obtained from a sample time of six consecutive minutes every two hours during a full $24 \mathrm{~h}$ day and they were used to: (1) calculate the distribution of the vehicles moving along the arteries with the respective bars of errors, and (2) to find out an adequate model, that turned to be essentially a hybrid one, which combines the open network model with the nonlinear one, both presented in Part I. This hybrid model is dynamical, i.e., it describes the time evolution of the traffic within the network continuously along a whole day. We have also compared the model - constructed from data of one single day - with the data obtained from different days. To our judgment this model is sound within the experimental errors, since it is able to describe satisfactorily 


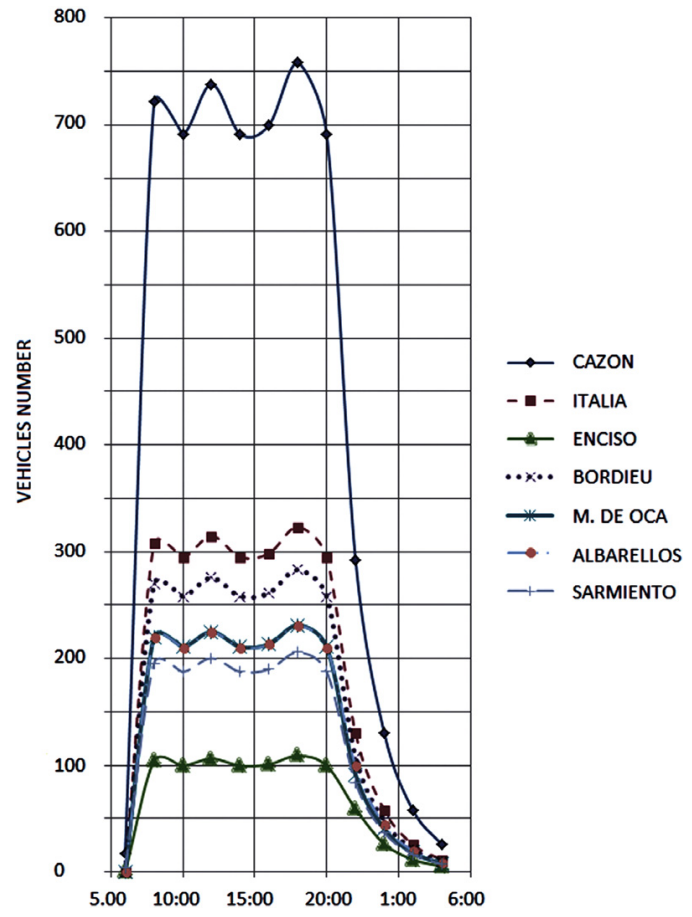

Fig. 11. The vertical axis represents the flux (number of vehicles during six minutes), according to the model, for several one-way arteries, or two-way as Cazón, Italia and Enciso.

the dynamical traffic trend. In order to optimize the traffic flow the model hinted for a change in the semaphores red light time duration. Finally, we estimate that the model is scalable, and as such it can be extended to larger networks.

\section{Acknowledgment}

SSM thanks the CNPq (Conselho Nacional de Desenvolvimento Científico e Tecnológico), Brazil, a Federal Brazilian Agency, for financial support.

\section{References}

[1] D. Otero, D. Galetti, S.S. Mizrahi, Physica A 509 (2018) 97-110.

[2] E. Manley, PLoS One 10 (5) (2015) e0127095.

[3] P.R. Bevington, D.K. Robinson, Data Reduction and Error Analysis for the Physical Sciences, third ed., McGraw Hill, New York, 2003, p. 67. 